A. YAMADA

KODAI MATH. J.

4 (1981), 266-277

\title{
ON MARDEN'S UNIVERSAL CONSTANT OF FUCHSIAN GROUPS
}

\author{
BY AKIRA YAMADA
}

Let $G$ be a Fuchsian group operating on the upper half plane $H$. For $z \in H$ and $r>0$, let $\Delta(z, r)$ be the open disc of radius $r$ and center $z$. Define $G^{z, r}$ to be the subgroup of $G$ generated by

$$
I(z, r)=\{g \in G ; d(z, g z)<2 r\}=\{g \in G ; \Delta(z, r) \cap \Delta(g z, r) \neq \phi\},
$$

where $d(\cdot, \cdot)$ is the hyperbolic distance induced by the Poincaré metric $|d z| / \operatorname{Im} z$.

In this paper all references to distance, lines, discs, etc., will be with respect to the hyperbolic geometry unless otherwise stated.

Marden [6] proved the following:

THEOREM. There is a constant $r>0$ such that, for any Fuchsian group $G$ and $z \in H$, the subgroup $G^{z, r}$ is either cyclic or infinte dihedral (i.e. is generated by two elliptic transformations of order 2).

Let $\mu(z, G)$ be the supremum of the set of constants $r$ satisfying the conclusion of the Theorem. In fact, this is the maximum by discreteness. Set

$$
\mu(G)=\inf _{z \in H} \mu(z, G) \text { and } \mu=\inf _{G} \mu(G) .
$$

$\mu$ will be called Marden's constant in this paper. The purpose of the paper is to determine Marden's constant explicitly. Our result is the following:

THEOREM 1. For any Fuchsian group $G$ we have

$$
\mu(G) \geqq \mu=\sinh ^{-1} \sqrt{\frac{4 \cos ^{2} \pi / 7-3}{8 \cos \pi / 7+7}}=0.131467 \cdots
$$

with equality occurring precisely when $G$ is the $(2,3,7)$ triangle group.

If we restrict ourselves to the case where $G$ is torsion-free, then much better bound is obtained.

THEOREM 2. For any torsion-free Fuchsian group $G$ we have

Received February 16, 1980 


$$
\mu(G) \geqq \sinh ^{-1} 1=\ln (1+\sqrt{2})=0.88137 \cdots .
$$

Equality occurs iff $G$ satisfies one of the following conditions:

1. $G$ is the $(\infty, \infty, \infty)$ triangle group.

2. $G$ has signature $(1 ; \infty)$ and is generated by two hyperbolic transformations with the same translation length $2 \sinh ^{-1} 1$.

Marden deduced his theorem from topological argument and a result of Purzitsky [9] on the classification of two-generator Fuchsian groups. On the other hand we give here an analytic proof of Theorem 1, which results from elementary but tedious calculations depending on repeated applications of Jørgensen's inequality [4]. Theorem 2 is obtained from straightforward applications of the results of Purzitsky [10] and Fricke-Klein [2]. For the convenience of the reader, however, we include the proof.

\section{Proof of Theorem 1 .}

1.1. For technical reasons, the proof will be divided into several cases according to the types of elements of $G$ (i.e. elliptic, parabolic and hyperbolic). We begin by considering the parabolic case. The following identity will be useful:

$$
\sinh \frac{1}{2} d\left(z, z^{\prime}\right)=\frac{\left|z-z^{\prime}\right|}{2 \sqrt{y y^{\prime}}}, \quad z=x+i y, z^{\prime}=x^{\prime}+i y^{\prime} .
$$

LEMMA 1. Let $A \in I(z, r)$ be paraboluc and assume that $r \leqq \ln \sqrt{2}=0.346 \cdots$. Then $G^{z, r}$ is a parabolic cyclic group.

Proof. It is no loss of generality to assume that $A=\left(\begin{array}{ll}1 & 1 \\ 0 & 1\end{array}\right)$ and that $G_{\infty}$, the stabilizer at $\infty$, is generated by $A$. Then $d(z, A z)<\ln 2$ implies $\operatorname{Im} z>\sqrt{2}$, so that $\Delta(z, r) \subset U=\{w ; \operatorname{Im} w>1\}$. But it is well-known that $U$ is precisely invariant under $G_{\infty}$ :

$$
\begin{cases}g U=U & \text { for every } g \text { in } G_{\infty}, \text { and } \\ g U \cap U=\phi & \text { for every } g \text { in } G \backslash G_{\infty} .\end{cases}
$$

Consequently, $G^{z, r}=G_{\infty}$ as desired.

1.2. For $A \in G$ and $B \in G$, set

$$
D(A, B)=\inf _{z \in H} \operatorname{Max}\left\{\sinh \frac{1}{2} d(z, A z), \sinh \frac{1}{2} d(z, B z)\right\} .
$$

Our task is to find lower bounds of $D(A, B)$ by using discreteness of $G$. Quantitative information of discrete groups is given by 
Jorgensen's inequality [4]: Let $\langle A, B\rangle$ be the group generated by two Möbıus transformations $A$ and $B$. If $\langle A, B\rangle$ is a non-elementary discrete group, then

$$
\left|\operatorname{tr}^{2} A-4\right|+|\operatorname{tr}[A, B]-2| \geqq 1
$$

where $[A, B]=A B A^{-1} B^{-1}$, the commutator of $A$ and $B$, and $\operatorname{tr} A$ denotes the trace of a matrix in $S L(2, C)$ representing $A$.

Let $A, B \in G$ be hyperbolic with distinct axes. By conjugation we may assume that the fixed points of $A$ (resp. $B$ ) are 0 and $\infty$ (resp. 1 and $\alpha \neq 1$ ). If Jørgensen's inequality is applied to $A$ and $B$, it follows easily that

$$
\operatorname{tr}[A, B]-2=\frac{16 \alpha}{(\alpha-1)^{2}} \sinh ^{2} \frac{l}{2} \sinh ^{2} \frac{m}{2}
$$

and

$$
4 \sinh ^{2} \frac{l}{2}\left(1+\frac{4|\alpha|}{(\alpha-1)^{2}} \sinh ^{2} \frac{m}{2}\right) \geqq 1
$$

where $l$ and $m$ are the translation lengths of $A$ and $B$ respectively. Interchanging $A$ with $B$, if necessary, we may assume that $l \leqq m$ in what follows.

1.2.1. Assume that the axes of $A$ and $B$ intersect simply. Then it is clear that $D(A, B)=\sinh m / 2$. Since $\alpha$ is negative, (1.2.3) implies

$$
4 \sinh ^{2} \frac{m}{2}\left(1+\sinh ^{2} \frac{m}{2}\right) \geqq 1 .
$$

Thus we obtain

LEMmA 2. If $A, B \in G$ are hyperbolic with their axes intersecting simply, then we have

$$
D(A, B) \geqq \sqrt{\frac{\sqrt{2}-1}{2}}=0.455 \cdots
$$

1.2.2. Assume that the axes of $A$ and $B$ do not intersect. In this case $\alpha$ is positive, since $\alpha \neq 0$ always by the fact that $G$ is Fuchsian. By magnification we can assume that $0<\alpha<1$. An easy calculation shows that the (Euclidian) line $\{w ; d(w, A w)=m\}$ intersects (or is tangent to) the axis of $B$ iff

$$
\sinh \frac{m}{2} \geqq \frac{1+\alpha}{1-\alpha} \sinh \frac{l}{2} \text {. }
$$

Now we divide the proof into two cases. If (1.2.4) is valid, then (1.2.3) gives

$$
\alpha\left(4 \sinh ^{2} \frac{m}{2}\right)^{2}+(1-\alpha)^{2} 4 \sinh ^{2} \frac{m}{2}-(1-\alpha)^{2} \geqq 0
$$

and therefore $D(A, B)>1 / 2$ since $D(A, B)=\sinh m / 2$. On the other hand if (1.2.4) 
does not hold, one verifies that $D(A, B)$ is given by $D(A, B)=\sinh t / 2$ where $t$ $(>m)$ is determined by requiring that the line $\{w ; d(w, A w)=t\}$ is tangent to the circle $\{w ; d(w, B w)=t\}$. After easy calculations we find

$$
\begin{aligned}
D^{2} & =\frac{4 \alpha \sinh ^{2} \frac{l}{2} \sinh ^{2} \frac{m}{2}}{4 \alpha \sinh ^{2} \frac{m}{2}-\left[(1+\alpha) \sinh \frac{m}{2}-(1-\alpha) \sinh \frac{l}{2}\right]^{2}} \\
& =\frac{4 \alpha \sinh \frac{l}{2} \sinh \frac{m}{2}}{2(1-\alpha)(1+\alpha)-(1-\alpha)^{2}\left(\sinh \frac{m}{2} / \sinh \frac{l}{2}+\sinh \frac{l}{2} / \sinh \frac{m}{2}\right)} .
\end{aligned}
$$

Thus,

$$
D^{2} \geqq \frac{1}{1-\alpha} \sinh \frac{l}{2} \sinh \frac{m}{2},
$$

while, by $l \leqq m,(1.2 .3)$ implies

$$
16 \alpha\left(\frac{1}{1-\alpha} \sinh \frac{l}{2} \sinh \frac{m}{2}\right)^{2}+4(1-\alpha)\left(\frac{1}{1-\alpha} \sinh \frac{l}{2} \sinh \frac{m}{2}\right) \geqq 1 .
$$

Hence it follows that $D \geqq 1 / 2$. Consequently, we have proved

LEMMA 3. Let $A, B \in G$ be hyperbolvc and assume that their axes do not intersect. Then we have

$$
D(A, B) \geqq \frac{1}{2}
$$

1.3. Let $A \in G$ be hyperbolic and let $B_{n} \in G$ be elliptic of order $n(n=2,3, \cdots)$. In the case of $n=2$, we assume that the fixed points of $B_{2}$ do not lie on the axis of $A$. Jørgensen's inequality is applicable to $A$ and $B_{n}(n=2,3, \cdots)$, since $\left\langle A, B_{n}\right\rangle$ is non-elementary. By conjugation we can assume that $A$ and $B_{n}$ have the following forms :

$$
A=\left(\begin{array}{rr}
\cosh \frac{l}{2} & e^{-c} \sinh \frac{l}{2} \\
e^{c} \sinh \frac{l}{2} & \cosh \frac{l}{2}
\end{array}\right), \quad B_{n}=\left(\begin{array}{rr}
\cos \frac{\pi}{n} & -\sin \frac{\pi}{n} \\
\sin \frac{\pi}{n} & \cos \frac{\pi}{n}
\end{array}\right) .
$$

Since $d\left(z, B_{n}^{k} z\right) \geqq d\left(z, B_{n} z\right)(1 \leqq k<n)$, this is legitimate. From $\operatorname{tr} A=2 \cosh l / 2$ and $\operatorname{tr}[A, B]-2=\left(\operatorname{tr}^{2} A-4\right) \cosh ^{2} c \sin ^{2} \pi / n$, Jørgensen's inequality yields

$$
4 \sinh ^{2} \frac{l}{2}\left(1+\cosh ^{2} c \sin ^{2} \frac{\pi}{n}\right) \geqq 1 .
$$

It is easy to see that the circle $\left\{w ; d\left(w, B_{n} w\right)=l\right\}$ intersects the axis of $A$ iff 


$$
\sinh \frac{l}{2} \geqq \sinh c \sin \frac{\pi}{n} .
$$

Again, we devide the proof into two cases.

1.3.1. If (1.3.2) is satisfied, then it is clear that $D\left(A, B_{n}\right)=\sinh l / 2$ and

$$
\cosh ^{2} c \sin ^{2} \frac{\pi}{n} \leqq 1+\sinh ^{2} \frac{l}{2} \text {. }
$$

From (1.3.1) we obtain

$$
D\left(A, B_{n}\right) \geqq \sqrt{\frac{\sqrt{5}-2}{2}}=0.343 \cdots .
$$

1.3.2. If (1.3.2) does not hold, then one verifies that

$$
D\left(A, B_{n}\right)=\sinh \frac{s_{n}}{2},
$$

where $s_{n}$ is determined by requiring that the circle $\left\{w ; d(w, A w)=s_{n}\right\}$ is tangent to the circle $\left\{w ; d\left(w, B_{n} w\right)=s_{n}\right\}$. We calculate $s_{n}$ and obtain

$$
\sinh ^{2} \frac{s_{n}}{2}=\frac{\cosh ^{2} c \sinh ^{2} \frac{l}{2}}{\left(\sin \frac{\pi}{n}+e^{c} \sinh \frac{l}{2}\right)\left(\sin \frac{\pi}{n}-e^{-c} \sinh \frac{l}{2}\right)} .
$$

From $\sin \pi / n \leqq \sin \pi / 2$, it is easily seen that we need only to consider the case $n=2$ under the condition that

$$
4 \sinh ^{2} \frac{l}{2}\left(1+\cosh ^{2} c\right) \geqq 1
$$

Thus,

$$
\begin{aligned}
\sinh ^{2} \frac{s_{2}}{2} & =\frac{\cosh ^{2} c}{\sinh ^{-2} \frac{l}{2}+2 \sinh c \sinh ^{-1} \frac{l}{2}-1} \\
& \geqq \frac{\cosh ^{2} c}{3+4 \cosh ^{2} c+4 \sqrt{\cosh ^{4} c-1}} \geqq \frac{1}{9},
\end{aligned}
$$

where in the last inequality the minimum $1 / 9$ is attained when $\cosh ^{2} c=5 / 3$. Therefore we have proved

LEMMA 4. Let $A \in G$ be hyperbolic and let $B \in G$ be elliptic of order $n$ ( $n=$ $2,3, \cdots)$, where, in the case of $n=2$, we assume that the fixed points of $B$ do not lie on the axis of $A$. Then we have $D(A, B) \geqq 1 / 3$.

1.4. Lemma 4 gives us information about the distribution of fixed points of elliptic transformations of order 2 in $G$ as follows (c. f. Corollary 1 of [6]). 
LEMMA 5. If $r<0.22$, then all the fixed points of elliptic transformations of order 2 belonging to $I(z, r)$ are colinear.

Proof. Let $A, B$ and $C \in I(z, r)$ be elliptic of order 2 with distinct fixed points $a, b$ and $c$ in $H$ respectively. Observe that $A B$ is hyperbolic with the axis connecting $a$ with $b$. The inequality

$$
d(z, A B z) \leqq d(z, A z)+d(z, B z)<4 r
$$

implies that $A B \in I(z, 2 r)$. Similarly, $A C \in I(z, 2 r)$. By Lemma 2 their axes must be the same, so that $a, b$ and $c$ are colinear.

1.5. We now come to the last and most important case where $A \in G$ and $B \in G$ are elliptic of order $m$ and $n, 2 \leqq m \leqq n, n \geqq 3$, respectively. For the estimate of $D(A, B)$, it is no loss of generality to assume that $A$ and $B$ are primitive, i. e. the angles of rotation are $2 \pi / m, 2 \pi / n$ resp.. Thus, we set

where

$$
A=r_{m}^{0}, \quad B=r_{n}^{c}, \quad c>0,
$$

$$
r_{n}^{c}=\left(\begin{array}{cc}
\cos \frac{\pi}{n} & -e^{-c} \sin \frac{\pi}{n} \\
e^{c} \sin \frac{\pi}{n} & \cos \frac{\pi}{n}
\end{array}\right) .
$$

$r_{n}^{c}$ is elliptic of order $n$ with a fixed point at $i e^{-c}$.

A constant $t$ is determined uniquely by requiring that the circle $\{w ; d(w, A w)$ $=t\}$ is tangent to the circle $\{w ; d(w, B w)=t\}$. Then it is seen that $D(A, B)=$ $\sinh t / 2$ and calculations give

$$
D(A, B)=\frac{\sinh ^{2} c \sin ^{2} \frac{\pi}{m} \sin ^{2} \frac{\pi}{n}}{\sin ^{2} \frac{\pi}{m}+\sin ^{2} \frac{\pi}{n}+2 \cosh c \sin \frac{\pi}{m} \sin \frac{\pi}{n}} .
$$

Observe that $D(A, B)$ is an increasing function of $c$, the hyperbolic distance between the fixed points of $A$ and $B$. To obtain a lower bound of $D$, we therefore need an estimate of $c$ in terms of the orders $m$ and $n$. Matelski [7] has found the lower bound of $c$ valid for any orders, which, however, is useless for our purpose. The desired bound can be obtained if we use the following Knapp's theorem.

Lemma 6 (Knapp [5]). Let $A, B$ be as in (1.5.1) and assume that $\langle A, B\rangle$ is discrete. If 


$$
\cosh c<\frac{1+\cos \frac{\pi}{m} \cos \frac{\pi}{n}}{\sin \frac{\pi}{m} \sin \frac{\pi}{n}}
$$

then $\langle A, B\rangle$ is a triangle group of Schwarz and $A B$ is elliptıc with $\operatorname{tr}(A B)=$ $-2 \cos \gamma$, where the pairs of $T=(m, n, \gamma)$ and the signature $S$ of $\langle A, B\rangle$ are given by one of the following:

1) $(T ; S)=(m, n, \pi / k ; m, n, k), 1 / m+1 / n+1 / k<1$,

2) $(T ; S)=(n, n, 2 \pi / k ; 2, n, k), 1 / n+1 / k<1 / 2,2 \nmid k$,

3) $(T ; S)=(2, n, 2 \pi / n ; 2,3, n), n \geqq 7,2 \nmid n$,

4) $(T ; S)=(3, n, 3 \pi / n ; 2,3, n), n \geqq 7,3 \nmid n$,

5) $(T ; S)=(n, n, 4 \pi / n ; 2,3, n), n \geqq 7,2 \nmid n$,

6) $(T ; S)=(3,7,2 \pi / 7 ; 2,3,7)$.

Note that, if the inequality (1.5.2) does not hold, we have

$$
D^{2} \geqq \frac{\left(\cos \frac{\pi}{m}+\cos \frac{\pi}{n}\right)^{2}}{2 \sin ^{2} \frac{\pi}{m}+2 \sin ^{2} \frac{\pi}{n}+\left(\cos \frac{\pi}{m}+\cos \frac{\pi}{n}\right)^{2}} \geqq \frac{1}{15}
$$

by monotonicity and $n \geqq 3$. On the other hand, using the identity

$$
\cos \gamma=\cosh c \sin \frac{\pi}{m} \sin \frac{\pi}{n}-\cos \frac{\pi}{m} \cos \frac{\pi}{n},
$$

we have

$$
D^{2}=\frac{\left(\cos \gamma+\cos \frac{\pi}{m} \cos \frac{\pi}{n}\right)^{2}-\sin ^{2} \frac{\pi}{m} \sin ^{2} \frac{\pi}{n}}{2\left(\cos \gamma+\cos \frac{\pi}{m} \cos \frac{\pi}{n}\right)^{2}+\sin ^{2} \frac{\pi}{m} \sin ^{2} \frac{\pi}{n}} .
$$

Observe that $D$ is monotone decreasing with respect to $\gamma, 1 / m, 1 / n$. Hence, to find the minimum of $D(A, B)$, Lemma 6 shows that we are only left with the following ten cases:

$$
\begin{aligned}
T= & (2,3, \pi / 7),(2,4, \pi / 5),(2,5, \pi / 4),(2,7, \pi / 3),(3,3,2 \pi / 7), \\
& (3,4, \pi / 3),(3,7, \pi / 2),(4,4,2 \pi / 5),(4,5, \pi / 2),(7,7,2 \pi / 3) .
\end{aligned}
$$

By direct calculations, we have

LEMma 7. Let $A \in G$ and $B \in G$ be elliptic of order $m$ and $n$ respectively with distinct fixed points. Assume that $2 \leqq m \leqq n$ and $n \geqq 3$. Then we have 


$$
D(A, B) \geqq \sqrt{\frac{4 \cos ^{2} \frac{\pi}{7}-3}{8 \cos \frac{\pi}{7}+7}}=0.13184 \cdots
$$

with equality occurnng precisely when $(m, n)=(2,3)$ and $\langle A, B\rangle$ is the $(2,3,7)$ triangle group.

1.6. Now we are ready to prove Theorem 1 . Set

$$
\mu=\sinh ^{-1} \sqrt{\frac{4 \cos ^{2} \frac{\pi}{7}-3}{8 \cos \frac{\pi}{7}+7}}
$$

and consider $I(z, \mu)$ for $z \in H$. By Lemma 1 we may assume that $I(z, \mu)$ does not contain any parabolic transformation. If there is a hyperbolic element $A \in$ $I(z, \mu)$, then all the axes of hyperbolic elements $I(z, \mu)$ coincide by Lemma 2 and Lemma 3. Moreover, by Lemma 4 we see that there is no elliptic element of order $\geqq 3$ in $I(z, \mu)$ and that all the fixed points of elliptic of order 2 , if these exist, lie on the axis of $A$. This implies that $G^{z, \mu}$ is either cyclic or infinite dihedral. In fact, if $P, Q \in I(z, \mu)$ are elliptic elements of order 2, then $P Q$ is hyperbolic with the same axis as $A$.

There remains to consider the case where $I(z, \mu)$ consists only of elliptic transformations. If $I(z, \mu)$ contains and elliptic element of order $\geqq 3$, then Lemma 7 shows that $G^{z, \mu}$ is cyclic. On the other hand, if $I(z, \mu)$ contains an elliptic element of order 2, Lemma 5 shows that $G^{z, \mu}$ is either cyclic or dihedral by a similar reasoning as above. From Lemma 7 it is seen that the constant $\mu$ is best possible. Since the $(2,3,7)$-group is a maximal Fuchsian group (see [3]), $G$ itself is the $(2,3,7)$-group if the subgroup $\langle A, B\rangle$ is. This completes the proof of Theorem 1.

\section{Proof of Theorem 2 .}

2.1. Let $\langle A, B\rangle$ be a doubly generated non-elementary subgroup of a torsionfree Fuchsian group $G$. Then Bers area theorem [1] gives

$$
2 g+b=3,
$$

where $g$ is the genus of $H /\langle A, B\rangle$ and $b$ is the number of its boundary components. Consequently, the possible signatures of $\langle A, B\rangle$ are the following:

$$
(0 ; \infty, \infty, \infty),(0 ; \infty, \infty ; 1),(0 ; \infty ; 2),(0 ;-; 3),(1 ; \infty),(1 ;-; 1) .
$$

In [10] Purzitsky gave necessary and sufficient conditions for $\langle A, B\rangle$ to be a Fuchsian group which is free. His results immediately yield inequalities which enable us to estimate $D(A, B)$ in torsion-free case. 
LEMma 8. Assume that $\langle A, B\rangle$ is a torsion-free non-elementary Fuchsian group. Then:

1) If $A$ and $B$ are hyperbolic with intersecting axes, we have

$$
\operatorname{tr}[A, B] \leqq-2 .
$$

Equality occurs iff the signature of $\langle A, B\rangle$ is $(1 ; \infty)$.

2) Otherwise, we have

$$
\operatorname{tr}[A, B] \geqq 18 .
$$

Equality occurs iff $\langle A, B\rangle$ is the $(\infty, \infty, \infty)$ triangle group.

Proof. For the proof of Case 1, the reader is referred to Theorem 8 in [10] of Purzitsky. We note that his proof remains valid under our hypothesis that $\langle A, B\rangle$ is torsion-free. Also, Case 2 is proved from his Theorems 2 , 4 , and 7 in [10] with the following additional observation. A Nielsen transformation is the replacement of $A$ and $B$ by new generators $A^{n} B$ and $A$ for some $n \in \boldsymbol{Z}$. In fact, he showed that, if $\langle A, B\rangle$ is torsion-free and Fuchsian, there is a pair of generators $A^{\prime}$ and $B^{\prime}$ obtained from repeated applications of Nielsen transformations to $A$ and $B$ such that

$$
\operatorname{tr} A^{\prime} \geqq 2, \quad \operatorname{tr} B^{\prime} \geqq 2 \text { and } \operatorname{tr} A^{\prime} B^{\prime} \leqq-2 .
$$

By mathematical induction one verifies easily that $\left[A^{\prime}, B^{\prime}\right]$ is conjugate to either $[A, B]$ or $[A, B]^{-1}$, and so that

$$
\operatorname{tr}[A, B]=\operatorname{tr}\left[A^{\prime}, B^{\prime}\right] \text {. }
$$

By Fricke's formula (see [2], p. 338)

$$
\operatorname{tr}[A, B]=\operatorname{tr}^{2} A+\operatorname{tr}^{2} B+\operatorname{tr}^{2} A B-\operatorname{tr} A \operatorname{tr} B \operatorname{tr} A B-2,
$$

it is seen from (2.1.1) and (2.1.2) that

$$
\operatorname{tr}[A, B] \geqq 18
$$

with equality occurring precisely when $\operatorname{tr} A^{\prime}=\operatorname{tr} B^{\prime}=-\operatorname{tr} A^{\prime} B^{\prime}=2$. Hence $\operatorname{tr}[A, B]$ $=18$ only if $\langle A, B\rangle=\left\langle A^{\prime}, B^{\prime}\right\rangle$ has signature $(0 ; \infty, \infty, \infty)$. On the other hand, the sufficiency follows from Nielsen's theorem on automorphisms of free groups [8] and the reasoning above.

2.2. Let $A$ and $B$ be parabolic with distinct fixed points. By conjugation it is no loss of generality to assume that

$$
A=\left(\begin{array}{ll}
1 & 1 \\
0 & 1
\end{array}\right), \quad B=\left(\begin{array}{ll}
1 & 0 \\
\lambda & 1
\end{array}\right), \quad \lambda>0 .
$$

We find easily that 


$$
D(A, B)=\sqrt{\lambda} / 2 \text { and } \operatorname{tr}[A, B]=2+\lambda^{2} .
$$

By Lemma 8 we have

Lemma 9. Let $A \in G$ and $B \in G$ be parabolic with distinct fixed points. Then we have $D(A, B) \geqq 1$ with equality occurring iff $\langle A, B\rangle$ is the $(\infty, \infty, \infty)$ trangle group.

2.3. Let $A$ be parabolic and let $B$ be hyperbolic. We may assume that $A$ and $B$ have the forms

$$
A=\left(\begin{array}{ll}
1 & \lambda \\
0 & 1
\end{array}\right), \quad B=\left(\begin{array}{cc}
\cosh \frac{l}{2} & \sinh \frac{l}{2} \\
\sinh \frac{l}{2} & \cosh \frac{l}{2}
\end{array}\right) \quad(\lambda, l>0),
$$

where $l$ is the translation length of $B$. Since $\operatorname{tr}[A, B]=2+\lambda^{2} \sinh ^{2} l / 2$, Lemma 8 implies

$$
\lambda \sinh \frac{l}{2} \geqq 4
$$

Now we shall treat separately the following two cases.

2.3.1. Assume that the line $\{w ; d(w, A w)=l\}$ intersects the axis of $B$. Then it is easy to see that

$$
D(A, B)=\sinh \frac{l}{2} \text { and } \sinh \frac{l}{2} \geqq \frac{\lambda}{2} .
$$

From (2.3.1) we obtain $D(A, B) \geqq \sqrt{2}$.

2.3.2. Contrarily, assume that the line does not intersect the axis of $B$. Then an easy calculation gives

$$
D(A, B)=\frac{\lambda \sinh \frac{l}{2}}{2 \sqrt{\lambda \sinh \frac{l}{2}-\sinh ^{2} \frac{l}{2}}}>\frac{1}{2} \sqrt{\lambda \sinh \frac{l}{2}} .
$$

From (2.3.1) we obtain $D(A, B)>1$. Consequently, we have proved

Lemma 10. Let $A \in G$ be parabolic and let $B \in G$ be hyperbolic. Then we have $D(A, B)>1$.

2.4. Let $A \in G$ and $B \in G$ be hyperbolic. As in section 1.2., we shall devide the proof into two cases according to the location of the axes. The results and notations in section 1.2 will be used freely in this section. 
2.4.1. Assume that the axes of $A$ and $B$ intersect simply. Set

$$
x=\operatorname{tr} A, \quad y=\operatorname{tr} B \quad \text { and } z=\operatorname{tr} A B .
$$

Then we know from Lemma 8 that

$$
x^{2}+y^{2}+z^{2}-x y z-2=\operatorname{tr}[A, B] \leqq-2,
$$

where $x=2 \cosh l / 2$ and $y=2 \cosh m / 2$. Now (2.4.1) gives

$$
\left(x^{2}-4\right)\left(y^{2}-4\right) \geqq 16 \text {, }
$$

which implies

$$
\operatorname{Max}\left\{x^{2}, y^{2}\right\} \geqq 8 \text {. }
$$

Since $D(A, B)=\operatorname{Max}\{\sinh l / 2, \sinh m / 2\}$, we have

$$
D^{2}(A, B)=\frac{1}{4}\left[\operatorname{Max}\left\{x^{2}, y^{2}\right\}-4\right] \geqq 1
$$

with equality occurring precisely when $\langle A, B\rangle$ has signature $(1 ; \infty)$ and $l=m=$ $2 \sinh ^{-1} 1$.

2.4.2. Assume that the axes of $A$ and $B$ do not intersect. From Lemma 8 and (1.2.2) we find

$$
\frac{\alpha}{(1-\alpha)^{2}} \sinh ^{2} \frac{l}{2} \sinh ^{2} \frac{m}{2} \geqq 1 \quad(0<\alpha<1, l \leqq m) .
$$

If the inequality (1.2.4) holds, then we have $D(A, B)=\sinh m / 2$, and therefore

$$
D^{4} \geqq \frac{(1+\alpha)^{2}}{\alpha}>4
$$

On the other hand, if (1.2.4) does not hold, then (1.2.5) and (2.4.2) show

$$
D^{4} \geqq \frac{1}{\alpha}>1 \text {. }
$$

Thus we have proved

LEMMA 11. Let $A \in G$ and $B \in G$ be hyperbolic with distinct axes. Then we have $D(A, B) \geqq 1$. Equality occurs iff $\langle A, B\rangle$ has signature $(1 ; \infty)$ and $l=m=$ $2 \sinh ^{-1} 1$. Here $l$ and $m$ are the translation lengths of $A$ and $B$ respectively.

2.5. Now we are ready to prove Theorem 2. In view of Lemmas $9-11$, it is clear that there remains only to prove the equality statement. For torsion-free Fuchsian groups, groups of signatures $(0 ; \infty, \infty, \infty)$ and $(1 ; \infty)$ are maximal with respect to inclusion relation. In fact, the area of a fundamental region of these groups is $2 \pi$, which is the minimum among the family of torsion-free Fuchsian groups. Thus the proof of Theorem 2 is completed. 
Remark. For surface groups (i. e. groups with compact quotient consisting of hyperbolic transformations alone), we clearly have $\mu(G)>\sinh ^{-1} 1$. Again, this inequality is best possible, which is seen by applying Klein's combination theorem to deform the groups with signature $(0 ; \infty, \infty, \infty)$ or $(1 ; \infty)$.

\section{REFERENCES}

[1] L. BERs, Inequalitıes for finıtely generated Kleınıan groups, J. d'Analyse Math. 18 (1967), 23-41.

[2] R. Fricke and F. Klein, Vorlesungen über die Theorie der automorphen Funktionen, Vol. 1, Leipzig, Teubner, 1897.

[3] L. Greenberg, Maximal Fuchsian groups, Bull. Amer. Math. Soc. 69 (1963), 569-573.

[4] T. Jørgensen, On discrete groups of Möbius transformations, Amer. J. Math. 98 (1976), 739-749.

[5] A.W. KNAPp, Doubly generated Fuchsian groups, Michigan Math. J. 15 (1968), 289-304.

[6] A. MARden, Universal properties of Fuchsian groups in the Poincare metric, Ann. of Math. Studies, 79 (1974), 315-339.

[7] J.P. Matelski, A compactness theorem for Fuchsian groups of the second kind, Duke Math. J. 43 (1976), 829-840.

[8] J. Nielsen, Die Isomorphismen der allgemeinen unendlichen Gruppe mit zweı Erzeugenden, Math. Ann. 78 (1918), 385-397.

[9] N. Purzitsky, All two-generator Fuchsian groups, Math. Z. 147 (1976), 87-92.

[10] N. Purzitsky, Two-generator discrete free products, Math. Z. 126 (1972), 209223.

Department of Mathematics

TOKYo Institute of TECHNOLOGY 